\title{
The role of adjuvant and neoadjuvant therapy in gastrointestinal stromal tumors
}

\author{
Margaret von Mehren \\ Department of Medical Oncology, Fox Chase Cancer Center, Philadelphia, USA
}

\begin{abstract}
Purpose of review-Gastrointestinal stromal tumors are the most common sarcoma of the gastrointestinal tract. A decade ago, the only therapy for gastrointestinal stromal tumors was surgery. Treatment paradigms changed with the discovery that gastrointestinal stromal tumor cells express KIT, a tyrosine kinase growth factor receptor, which is mutated in $85 \%$ of cases. Imatinib and sunitinib are tyrosine kinase inhibitors with activity against advanced gastrointestinal stromal tumors. This review will discuss the available data on the use of imatinib in the adjuvant setting and the role of imatinib and sunitinib in the neoadjuvant setting.
\end{abstract}

Recent findings-Retrospective series and prospective studies have demonstrated the benefit of adjuvant imatinib. Randomized data show improved recurrence free survival in patients receiving imatinib for 1 year postoperatively. Ongoing studies are further defining the length of adjuvant therapy. The neoadjuvant treatment decreases tumor size to allow for surgical resection with less morbidity. The imatinib neoadjuvant therapy in a prospective randomized study was safe with encouraging outcomes. This approach for palliating advanced disease also appears to be safe following imatinib, sunitinib, or other tyrosine kinase inhibitors therapy.

Summary-Treatment for gastrointestinal stromal tumors, formerly limited to surgery, now is a combination of surgery and tyrosine kinase inhibitors therapy. Combination therapy is safe and improves outcomes, particularly in the adjuvant setting.

\section{Keywords}

adjuvant; gastrointestinal stromal tumors; imatinib; neoadjuvant; sunitinib

\section{Introduction}

Gastrointestinal stromal tumors (GISTs) were recognized as distinct gastrointestinal mesenchymal tumor apart from leiomyosarcomas and leiomyomas by Mazur and Clark [1] because they had both neural and smooth muscle features. The discovery by Hirota and colleagues [2] further distinguished that GISTs expressed KIT, a cell surface growth factor receptor and proto-oncogene. KIT was found in many cases to be mutated and thus constitutively activated. Indeed, tumors developed in a knock-in mouse model of a GISTderived mutated KIT.

Understanding the biology of GIST was clinically significant because it provided a therapeutic target. Before the use of tyrosine kinase inhibitors (TKI), surgery was the mainstay of therapy as chemotherapy and radiation therapy had limited efficacy [3]. Retrospective series suggested that patients with completely resected primary tumors had a

Correspondence to Margaret von Mehren, Department of Medical Oncology, Fox Chase Cancer Center, Philadelphia, PA 19111, USA Tel: +1 215728 2674; fax: +1 215728 3639; Margaret.vonMehren@fccc.edu. 
survival of approximately 8 years, but fell to only 2 years if not completely resected [4]. Outcomes were inferior for patients who had locally recurrent and metastatic disease with all patients eventually succumbing to their disease.

Imatinib, a TKI with efficacy against KIT, PDGFR, and Abl/Bcr-Abl, demonstrated significant efficacy in patients with advanced GISTs. Up to $85 \%$ of the patients treated with imatinib had long-term disease stabilization as well as partial responses, with a minority $(<10 \%)$ achieving complete responses [5-7]. Sunitinib, a second TKI that is effective against KIT, PDGFR and vascular endothelial growth factor receptors (VEGFR), has also been shown to have efficacy in palliating disease in patients with tumors that have progressed on imatinib [8]. Given the activity of TKI in the metastatic disease setting, investigators have sought to test their utility in the adjuvant and neoadjuvant setting. The rationale for these approaches as well as the reported efficacy is discussed in this review.

\section{Adjuvant therapy}

Tyrosine kinase inhibition in the metastatic disease setting is effective and leads to a large proportion of patients have their disease palliated for many years. In the primary setting, surgery is effective, however many patients ultimately recur and die of their disease. In retrospective series, half of the patients will have local or distant recurrences with 42-54\% surviving 5 years $[4,9]$. Factors associated with a poorer outcome with surgery alone include: tumors with five or more mitoses per 50 high power field, size $10 \mathrm{~cm}$ or greater, primary tumors of the small bowel, evidence of perforation, and incomplete primary tumor resection $\left[10^{\circ}, 11\right]$.

The first study to assess the efficacy of imatinib in the adjuvant setting was the American College of Surgical Oncology Group (ACOSOG) study Z9000, a phase II trial of low-dose imatinib for 12 months in patients with resected GISTs at a high risk for disease recurrence because of a large primary tumor, tumor rupture, or evidence of a limited number of peritoneal metastases. Adjuvant imatinib was found to be safe without any unanticipated toxicity [12]. Outcomes from this study were recently reported [13*]. At a median follow-up of 4 years, the 1,2, and 3-year overall survival (OS) rates were 99, 97, and 97\%, respectively; the median survival for a comparable group of patients in retrospective series was 2 years [4]. The 1,2, and 3-year recurrence free survival (RFS) rates in the Z9000 study were 94,73 , and $61 \%$, respectively. These data suggest that imatinib can improve RFS and OS in this group of patients compared with historical controls.

Nilsson and colleagues $\left[14^{\circ}\right]$ reported on 23 consecutive patients treated with adjuvant imatinib, $400 \mathrm{mg}$ daily for 1 year. All the patients had high-risk tumors, with a median tumor size of $9.4 \mathrm{~cm}$, which were completely resected. KIT and PDGFRA mutational analysis was performed with 17 KIT exon 11 mutations, one KIT exon 9 mutation, one PDGFR exon 18 mutations, and four tumors without KIT or PDGFR mutations. They compared the outcomes in the adjuvant patients to 48 historical controls. The historical controls had a slightly larger mean tumor size of $12.3 \mathrm{~cm}$, with 29 KIT exon 11 mutations, one each PDGFR exon 12 and 18 mutations, and the remainder without mutations. They determined a statistically significant improvement in outcome after a mean follow-up of 3 years, with only 1 of 23 patients in the adjuvant group having relapsed compared with 32 of 48 patients without treatment $(P=0.001)$. The patient that recurred in the adjuvant cohort was a pediatric GIST patient whose tumor did not have detectable mutation. This report clearly suggests that there is a benefit to adjuvant therapy; however, the control group may have had a poorer outcome given the slightly larger size of the tumors and the increased number of patients with mutations with an inherently poorer outcome. 
The results of ACOSOG Z9001, a prospective randomized study [15*0] of adjuvant imatinib in contrast with placebo following complete surgical resection of primary GISTs greater than $3 \mathrm{~cm}$ in size, has been reported. The placebo control arm provides an appropriate control; the outcome of GIST following resection utilizing modern surgical techniques and surveillance strategies. This study demonstrated an improved RFS for patients receiving adjuvant imatinib, however with no difference in OS between these two patient groups to date. The difference in RFS, however, appeared to be most significant for those with tumors greater than $10 \mathrm{~cm}$ (hazard ratio $=0.19, P<0.001$ ), less so for those with tumors $6-10 \mathrm{~cm}$ (hazard ratio $=0.37, P=0.01$ ), but was not significant in those with the smallest tumors 3-6 $\mathrm{cm}$ (hazard ratio $=0.44, P=0.15$ ) $\left[15^{\circ}\right]$. There are ongoing studies in Europe that will add to our understanding of adjuvant imatinib. The Scandinavian Sarcoma Group is randomizing patients at high risk of recurrence to low-dose imatinib for 12 versus 36 months; they have accrued 345 of a planned 400 patients. The European Organization for Research and Treatment of Cancer (EORTC) is testing low-dose imatinib (400 mg daily) for 24 months in contrast with placebo in this same population. The EORTC trial is the only study whose primary endpoint is overall survival rather than progression free survival. As such, information on the outcomes of this trial is likely to take many years because patients are palliated so well by the current TKI therapy.

The National Comprehensive Cancer Center Network (NCCN) treatment guidelines for adjuvant therapy in GISTs have been updated and indicate that adjuvant imatinib can be considered postoperatively. At this time, the data supports 1 year of imatinib therapy, however this may change with the results of ongoing trials. The recommendations from the consensus meeting of GISTs sponsored by the European Society of Medical Oncology recommends adjuvant therapy only in the setting of a clinical trial [16].

\section{Neoadjuvant therapy}

The role of neoadjuvant therapy is to decrease the size of a mass in an attempt to completely resect a primary GIST. This approach has been recommended in NCCN guidelines for tumors that would be considered respectable, but at a high risk of postoperative morbidity. In such cases, treatment with imatinib to maximal response followed by surgical resection is an appropriate option for patients. Close follow-up is required to ensure that patients are responding to the therapy and do not lose the opportunity to undergo a potentially curative resection.

Several studies have been conducted evaluating this approach. Trent and colleagues evaluated the role of preoperative imatinib in patients scheduled to undergo GIST resections in a study designed to ask biologic questions. They hypothesized that the earliest changes induced by imatinib were apoptosis and changes in vascularity. They performed imaging studies and correlated their findings to changes observed in preimatinib and postimatinib tissue samples. Patients received imatinib a dose of $600 \mathrm{mg}$ daily. Perfusion computed tomography and fluoro deoxy-glucose (FDG) PET studies were performed at baseline and then after 3,5, or 7 days of therapy. The perfusion studies assessed blood flow and blood volumes of the entire tumor. Pet scans measured the standardized uptake value of FDG. The pretreatment biopsies and samples from surgical resections were studied to quantify endothelial and tumor cell apoptosis, micro vessel density, as well as the presence or absence of phosphorylation of KIT and PDGFR kinases. They found that seven of 10 patients had a decrease in standardized uptake value (SUV) of greater than $30 \%$ whereas three had no decrease or no baseline FDG avidity. Those with decreases in the SUV also had a decrease in tumor blood flow and blood volume whereas those with no change in SUV had an increase in blood flow and blood volume ( -37.8 vs. $+38.5 \%, P=0.02$ and -29.5 vs. $+24 \%, P=0.04$, respectively). Tumors that had a decrease in phosphorylation of KIT in the 
endothelial cells and tumor cells were noted to have more endothelial and tumor cell death compared with those samples without these changes in phosphorylation. Tumor blood flow or blood volume decreases correlated with increased endothelial cell death. Tumors with the greatest changes in microvessel density were those with the greatest decreases in FDG uptake. These studies led the authors to conclude that imatinib can induce tumor cell apoptosis and may have antivascular effects on GISTs. This study answered interesting biologic questions, but did not provide much clinical information on this approach given the short course of imatinib preoperatively.

The Radiation Oncology Therapy Group (RTOG) study S0132 evaluated the safety and efficacy of 8 weeks of preoperative imatinib followed by surgical resection for patients with primary resectable or advanced disease that was deemed to be completely respectable by a surgical oncologist at the initial evaluation for the study $\left[17^{\circ}\right]$. Patients with primary disease received 2 years of adjuvant imatinib, whereas those with metastatic disease remained on imatinib until evidence of disease progression. Imatinib was given at an initial dose of 600 mg daily. Patients were monitored closely with CT and PET scans at baseline, following 1 week of therapy, at 4 weeks, and 8 weeks of therapy. In these studies, patients who had progressive disease were removed from the study, whereas all others received surgery. Fiftytwo patients of the 63 enrolled are analyzable for toxicity and operative outcomes. R0 resection (complete gross tumor removal) was performed in 77\%, R1 (resection leaving minimal residual disease) in 15\%, and R2 (resection leaving gross residual disease) in $8 \%$ of patients with locally advanced tumors. In patients with recurrent or metastatic GIST, R0 resection was performed in 58\%, R1 in 5\%, R2 in 32\%, with one patient's outcome unknown. The operative complications included one septic death 2 months following the surgery, wound infection $(7 \%)$, hemorrhage $(4 \%)$, respiratory complications $(11 \%)$, cardiac complications (7\%), anastomotic disruption (2\%), and abscess (4\%). Re-initiation of imatinib postoperatively was delayed due to surgical complications in $16 \%$. At 2 years, patients with locally advanced disease have a progression free survival (PFS) of $82 \%$ (95\% confidence interval (CI) 68,97), and an OS of $93 \%(84,100)$. Patients with recurrent or metastatic disease have a PFS of $73 \%(54,91)$, and an OS of $91 \%(79,100)$. This study also obtained pretreatment biopsies and specimens from the surgical resection to determine the mutation status of the tumor, assess markers of glucose metabolism as they correlate to PET response, as well as to conduct gene expression arrays to determine a signature of response to imatinib. These data have yet to be reported. This study demonstrates that neoadjuvant therapy can be given safely, and along with the study by Trent and colleagues demonstrates that biologic studies can be conducted to assess the effects of imatinib (or other agents) on GISTs.

Clinically, another important role for neoadjuvant therapy is in those patients who have advanced unresectable disease requiring palliation. Many patients will develop resistance to imatinib and subsequent therapies. Resistance often presents as an outgrowth of one tumor cell clone that has additional genetic changes. Therefore, the role of resection to control a limited number of progressing lesions in the setting of an otherwise stable disease is of interest. Several series have been published demonstrating the safety of this approach following therapy with imatinib or sunitinib $\left[18,19,20^{\circ}\right]$. Multiple types of surgical resections, simple as well as complicated, have been performed following therapy with imatinib, sunitinib, and other tyrosine kinase inhibitors without unacceptable postoperative morbidity. Although sunitinib targets VEGFR, increased risk of bleeding, prolonged wound healing, or dehiscence does not appear to be excessive. For patients that undergo a complete surgical resection in the setting of metastatic disease, discontinuation of therapy is not recommended because of short-interval progression as demonstrated by the BFR14 study $\left[21^{\circ}\right]$. Lessons from these series also suggest that surgical resection in a patient without clinical response to current management in whom alternative therapies are not available is 
likely to lead to a short interval until tumor recurrence. Raut and colleagues found that the disease state at the time of surgery was an important predictor of the ability to perform a complete resection, the 12-month PFS and OS [18]. Patients with stable disease, limited disease progression, or diffuse disease progression had a 12-month PFS of 80,33 , and $0 \%$, respectively. These same trends were echoed in the 12-month OS of 95,86 and $0 \%$.

\section{Conclusion}

Treatment for GISTs has become increasingly complex, yet with this complexity has come increased therapeutic efficacy. Prior to the development of TKI therapy, the only effective therapy was surgery. In the primary disease setting, half recurred and with recurrence the chance for cure vanished. Early results of the(Please delete the) adjuvant therapy suggest benefit from the use of imatinib, especially in tumors that are large in size. Therapy with imatinib increases the RFS rate. What is not known at this time is if imatinib only delays tumors from recurring, or if treatment is curing some patients with GISTs. To date, there is limited information on the benefit of adjuvant therapy for GISTs with different KIT and PDGFR mutations; one might anticipate that the benefit of imatinib will be greatest in tumors with KIT exon 11 mutations and less so for other types of mutations, as has been noted in the advanced metastatic disease setting [22-24]. In addition, there may be a benefit to high-dose imatinib (400 $\mathrm{mg}$ twice daily) in patients whose GISTs contain an exon nine mutations, as has been demonstrated in the metastatic disease setting [23]. Further analysis of data from the adjuvant studies should provide an additional insight to this topic.

Neoadjuvant therapy is safe and can be a useful adjunct to resection both in the primary disease setting as well as in the advanced setting. For patients with advanced disease, surgical resection in the setting of TKI therapy must be balanced by disease status and the availability of effective therapy given there can be rapid progression and limited PFS and OS for those without an effective treatment option.

\section{Acknowledgments}

The present work is supported in part by NIH grant (CA106588).

\section{References and recommended reading}

Papers of particular interest, published within the annual period of review, have been highlighted as:

-of special interest

•• of outstanding interest

1. Mazur MT, Clark HB. Gastric stromal tumors: reappraisal of histiogenesis. Am J Surg Pathol 1983;7:507-519. [PubMed: 6625048]

2. Hirota S, Isozaki K, Moriyama Y, et al. Gain-of-function mutations of $c$-kit in human gastrointestinal stromal tumors. Science 1998;279:577-580. [PubMed: 9438854]

3. von Mehren M, Watson JC. Gastrointestinal stromal tumors. Hematol Oncol Clin North Am 2005;19:547-564. vii. [PubMed: 15939196]

4. DeMatteo RP, Lewis JJ, Leung D, et al. Two hundred gastrointestinal stromal tumors: recurrence patterns and prognostic factors for survival. Ann Surg 2000;231:51-58. [PubMed: 10636102]

5. Demetri G, vonMehren M, Blanke C, et al. Efficacy and safety of imatinib mesylate in advanced gastrointestinal stromal tumors. N Engl J Med 2002;347:472-480. [PubMed: 12181401] 
6. van Oosterom A, Judson I, Verweij J, et al. Update of phase I study of imatinib (STI571) in advanced soft tissue sarcomas and gastrointestinal stromal tumors: a report of the EORTC Soft Tissue and Bone Sarcoma Group. Eur J Cancer 2002;38:S83-87. [PubMed: 12528778]

7. Verweij J, Casali PG, Zalcberg J, et al. Progression-free survival in gastrointestinal stromal tumours with high-dose imatinib: randomized trial. Lancet 2004;364:1127-1134. [PubMed: 15451219]

8. Demetri GD, van Oosterom AT, Garrett CR, et al. Efficacy and safety of sunitinib in patients with advanced gastrointestinal stromal tumour after failure of imatinib: a randomized controlled trial. Lancet 2006;368:1329-1338. [PubMed: 17046465]

9. Pierie J, Choudry U, Muzikansky A, et al. The effect of surgery and grade on outcome of gastrointestinal stromal tumors. Arch Surg 2001;136:383-389. [PubMed: 11296107]

10•. DeMatteo R, Gold J, Saran L, et al. Tumor mitotic rate, size and location independently predict recurrence after resection of primary gastrointestinal stromal tumor (GIST). Cancer 2008;112:608-615. Single institution retrospective analysis of tumor characteristics associated with recurrence after initial primary GIST resection. [PubMed: 18076015]

11. Eisenberg B, Judson I. Surgery and Imatinib in the Management of GIST: emerging approaches to adjuvant and neoadjuvant therapy. Ann Surg Oncol 2004;11:465-474. [PubMed: 15123459]

12. Dematteo R, Antonescu C, Chadaram V, et al. Adjuvant imatinib mesylate in patients with primary high risk gastrointestinal stromal tumor (GIST) following complete resection: safety results from the U.S. Intergroup Phase II trial ACOSOG Z9000. Am Soc Clin Oncol 2005;23:9009.

13•. DeMatteo, R.; Owzar, K.; Antonescu, C., et al. Efficacy of adjuvant imatinib mesylate following complete resection of localized, primary gastrointestinal stromal tumor (GIST) at high risk of recurrence: the U.S. Intergroup phase II trial ACOSOG Z9000; Orlando. American Society of Clinical Oncology Gastrointestinal Cancer Symposium; Florida: 2008. First report of the therapeutic outcomes of ACOSOG Z9000, a nonrandomized trial of adjuvant imatinib in patients with resected high-risk GIST

14-. Nilsson B, Sjolund K, Kindblom L-G, et al. Adjuvant imatinib treatment improves recurrence-free survival in patients with high-risk gastrointestinal stromal tumours (GIST). Br J Cancer 2007;96:1656-1658. Single institution experience with adjuvant imatinib compared with a historical control. This is the first report that also includes data on the mutation status of each tumor. [PubMed: 17533389]

15•.. DeMatteo R, Owzar K, Maki R, et al. Adjuvant imatinib mesylate increases recurrence free survival (RFS) in patients with completely resected localized primary gastrointestinal stromal tumor (GIST): North American Intergroup Phase III trial ACOSOG Z9001. J Clin Oncol 2007;25:A10079. Initial presentation of ACOSG Z9001, a randomized prospective double blind placebo controlled trial of imatinib in the adjuvant setting demonstrating improved recurrence free survival in patients with GIST larger than $6 \mathrm{~cm}$ in size.

16. Blay J-Y, Bonvalot S, Casali P, et al. Consensus meeting for the management of gastrointestinal stromal tumors: report of the GIST consensus conference of 20-21 March 2004, under the auspices of ESMO. Ann Oncol 2004;16:566-578. [PubMed: 15781488]

17•. Eisenberg, B.; Harris, J.; Blanke, C., et al. Phase II trial of neoadjuvant/adjuvant imatinib mesylate (im) for advanced primary and recurrent operable gi stromal tumor (GIST) - early results of RTOG 0132. Surgical Society of Oncology; Chicago, IL: 2008. First abstract presenting the data from RTOG S0132, a prospective trial of neoadjuvant and adjuvant imatinib in patients with locally advanced, recurrent or metastatic disease that was deemed completely resectable at initial evaluation

18. Raut CP, Posner M, Desai J, et al. Surgical management of advanced gastrointestinal stromal tumors after treatment with targeted systemic therapy using kinase inhibitors. J Clin Oncol 2006;24:2325-2331. [PubMed: 16710031]

19. Hohenberger P, Langer C, Pistorius F, et al. Indication and results of surgery following imatinib treatment of locally advanced or metastatic GI stromal tumors (GIST). J Clin Oncol 2006;24:9500.

20•. Andtbacka R, Ng C, Scaife C, et al. Surgical resection of gastrointestinal stromal tumors after treatment with imatinib. Ann Surg Oncol 2007;14:14-24. Single institution retrospective series of surgical outcomes following imatinib therapy in the locally advanced and metastatic disease setting. [PubMed: 17072676] 
21 • Blay JY, Le Cesne A, Ray-Coquard I, et al. Prospective multicentric randomized phase III study of imatinib in patients with advanced gastrointestinal stromal tumors comparing interruption versus continuation of treatment beyond 1 year: the French Sarcoma Group. J Clin Oncol 2007;25:1107-1113. Prospective randomized trial demonstrating rapid recurrence of GISTs in patients treated with advanced imatinib for 1 year followed by discontinuation, even in the setting of complete surgical resection. [PubMed: 17369574]

22. Debiec-Rychter M, Dumez H, Judson I, et al. Use of c-KIT/PDGFRA mutational analysis to predict the clinical response to imatinib in patients with advanced gastrointestinal stromal tumours entered on phase I and II studies of the EORTC Soft Tissue and Bone Sarcoma Group. Eur J Cancer 2004;40:689-695. [PubMed: 15010069]

23. Debiec-Rychter M, Sciot R, Le Cesne A, et al. KIT mutations and dose selection for imatinib in patients with advanced gastrointestinal stromal tumours. Eur J Cancer 2006;42:1093-1103. [PubMed: 16624552]

24. Heinrich M, Corless C, Demetri G, et al. Kinase mutations and imatinib mesylate response in patients with metastatic gastrointestinal stromal tumor. J Clin Oncol 2003;21:4342-4349. [PubMed: 14645423] 